\title{
Overview of flavor physics results at Belle
}

\author{
Yosuke Yusa ${ }^{1, a}$ \\ ${ }^{1}$ Niigata University, Niigata 950-2181
}

\begin{abstract}
We will present recent topics of the flavor physics in decays of the heavy mesons at the Belle experiment. Most of the results are based on a full data set which corresponds to 772 million $B \bar{B}$ pairs collected at the $\Upsilon(4 S)$ resonance with the Belle detector at the KEKB asymmetric energy $e^{+} e^{-}$collider. We also discuss about prospects of the studies in the future $B$-factory experiment.
\end{abstract}

\section{Introduction}

Measurements of various $B$ meson decays induced by interactions of flavor such as $C P$ violations, decay rates with high precision give hints to the new physics beyond the Standard Model (SM). To investigate the difference between several decay modes or deviation from theoretical predictions in the SM with a large statistic data sample will be a good probe to find a tiny effect from the new physics. In this scheme, we can access to the phenomena that appears in a very high energy scale and this analysis scheme is so called a luminosity frontier. In this report, we review recent progress in the Belle experiment.

The results reported herein are based on a data sample containing $772 \times 10^{6} B \bar{B}$ pairs collected at the $\Upsilon(4 S)$ resonance with the Belle detector [1] at the KEKB asymmetric energy $e^{+} e^{-}$(3.5 on $8.0 \mathrm{GeV}$ ) collider [2]. The Belle detector consists of six types of sub-detectors: a silicon vertex detector (SVD), a 50-layer central drift chamber (CDC), an array of aerogel threshold Cherenkov counters (ACC), a barrel-like arrangement of time-of-flight scintillation counters (TOF), a CsI(Tl) crystal-based electromagnetic calorimeter (ECL), and a multilayer structure of resistive plate counters and iron plates to detect $K_{L}$ mesons and muons (KLM). All the sub-detectors except for the KLM are located inside a $1.5 \mathrm{~T}$ solenoidal magnetic field. Two inner detector configurations were used: a 2.0 $\mathrm{cm}$ beampipe and a three-layer SVD were used for the first sample of $152 \times 10^{6} B \bar{B}$ pairs, while a 1.5 $\mathrm{cm}$ beampipe, a four-layer SVD and a small-cell CDC were used to record the remaining $620 \times 10^{6} B \bar{B}$ events [3]. The latter sample has been reprocessed with an improved track reconstruction algorithm, which significantly increased the signal reconstruction efficiency especially in the decay modes in which low momentum charged particles are appeared in the final states.

Using information collected with the Belle detector such as particle momentum, charge, energy and so on, we reconstruct the $B^{0}$ meson with decay products. We identify $B$ candidates using two kinematic variables: the beam-energy constrained mass, $M_{\mathrm{bc}} \equiv \sqrt{E_{\text {beam }}^{2}-\left|\sum_{i} \vec{p}_{i}\right|^{2}}$, and the energy difference, $\Delta E \equiv \sum_{i} E_{i}-E_{\text {beam }}$, where $E_{\text {beam }}$ is the beam energy, and $\vec{p}_{i}$ and $E_{i}$ are the momentum and

\footnotetext{
ae-mail: yusa@hep.sc.niigata-u.ac.jp
} 
energy, respectively, of the $i$-th daughter of the reconstructed $B$ candidate in the $e^{+} e^{-}$center-of-mass (CM) frame. Signal events typically peak at the nominal $B$-meson mass for $M_{\mathrm{bc}}$ and at zero for $\Delta E$. The dominant background arises from the $e^{+} e^{-} \rightarrow q \bar{q}$ continuum process, where $q$ denotes $u, d, s$ or $c$. To suppress this background, we employ continuum suppression variables calculated from shape variables of the $B$ decays. We finally perform a fit with these variables to extract target physics parameters in each decay mode to enhance the signal reconstruction efficiency and background rejection.

\section{CP Violation}

Measurements of the $C P$ violation in the $B$ meson decays are one of the main target in the $B$-factory experiment and they achieve great breakthrough in measurements of the parameters such as angles of an unitary triangle of the Cabbibo-Kobayashi-Maskawa (CKM) matrix which describe transition in the quark sector. In the Kobayashi-Maskawa theory [4] in which the quark-mixing matrix has a single irreducible complex phase that gives rise to all $C P$-violating asymmetries. In the decay sequences $\Upsilon(4 S) \rightarrow B^{0} \bar{B}^{0} \rightarrow f_{C P} f_{\text {tag }}$, where one of the $B$ mesons decays at time $t_{C P}$ to a $C P$ eigenstate $f_{C P}$ and the other decays at time $t_{\text {tag }}$ to a final state $f_{\text {tag }}$ that distinguishes between $B^{0}$ and $\bar{B}^{0}$, the decay rate has a time dependence given by

$$
P(\Delta t, q)=\frac{e^{-|\Delta t| / \tau_{B^{0}}}}{4 \tau_{B^{0}}}\left[1+q \cdot\left[S_{f} \sin \left(\Delta m_{d} \Delta t\right)+A_{f} \cos \left(\Delta m_{d} \Delta t\right)\right]\right] .
$$

Here, $S_{f}$ and $A_{f}$ are parameters that describe mixing-induced and direct $C P$ violation, respectively, $\tau_{B^{0}}$ is the $B^{0}$ lifetime, $\Delta m_{d}$ is the mass difference between the two $B^{0}$ mass eigenstates, $\Delta t=t_{C P}-t_{\mathrm{tag}}$, and the $b$-flavor charge, $q=+1(-1)$ when the tagged $B$ meson is a $B^{0}\left(\bar{B}^{0}\right)$. At the KEKB asymmetricenergy $e^{+} e^{-}$collider [2], $\Upsilon(4 S)$ is produced with a Lorentz boost. Since $B^{0}$ and $\bar{B}^{0}$ mesons are approximately at rest in the $\Upsilon(4 S)$ center-of-mass system (CM), the $\Delta t$ can be determined from the displacement between the $f_{C P}$ and $f_{\text {tag }}$ decay vertices along flight direction of $\Upsilon(4 S), z: \Delta t \simeq\left(z_{C P}-\right.$ $\left.z_{\text {tag }}\right) /(\beta \gamma c) \equiv \Delta z /(\beta \gamma c)$. Since most of the $C P$ eigenstates are not flavor-specific, $q$ is determined using the information of $f_{\text {tag }}$ such as flavor of charged lepton, high momentum kaon and low momentum pion from $D^{*}$ decay.

Decays $B^{0} \rightarrow(c \bar{c}) K^{0}$ induced by the $b \rightarrow c \bar{c} s$ quark transition are referred to as "golden modes" since these are clean both experimentally and theoretically due to relatively large branching fractions and small background contamination [5]. A color-suppressed tree diagram is a dominant contribution of these mode and $S_{f}=-\xi_{f} \sin 2 \phi_{1}$ is predicted in a good approximation in the SM, where $\xi_{f}=$ $+1(-1)$ corresponds to $C P$-even (-odd) final states and $\phi_{1}$ is an angle of the unitary triangle defined using CKM matrix elements: $\phi_{1}=\arg \left(V_{c d} V_{c b}^{*} / V_{t d} V_{t b}^{*}\right)$. On the other hand, decays induced by the $b \rightarrow s q \bar{q}(q=u, d s)$ transitions are also th $C P$-eigenstates of the $\phi_{1}$ but the Feynman diagram so called "penguin diagram" contains a loop so that they are sensitive to the contribution of the new physics. Consequently, search for the extra- $C P$ phase from the new physics is done through the precise measurement of the $p h i_{1}$ in several $B$ decays.

Among such decays, we recently release the new result in the $B^{0} \rightarrow \omega K^{0}$ decay [6]. Figure 1 and 2 are the reconstructed kinematic variables and decay time difference distributions, respectively. From these, we obtain the result $\sin 2 \phi_{1}=0.91 \pm 0.32 \pm 0.06$. The statistical uncertainty is still large but evidence of the $C P$-violation is seen with the significance of $3.1 \sigma$. This is consistent with the SM expectation from the precise measurement in decays $B^{0} \rightarrow(c \bar{c}) K^{0}$ and other decay modes which is induced by the $b \rightarrow s q \bar{q}$ penguin process.

$\phi_{2}\left(=\arg \left(V_{u d} V_{u b}^{*} / V_{t d} V_{t b}^{*}\right)\right)$ is measured using the decays which induced by $b \rightarrow u \bar{u} d$ tree diagram. However, there is contamination from an extra-CP phase in the measured variable due to the $b \rightarrow u \bar{u} d$ 

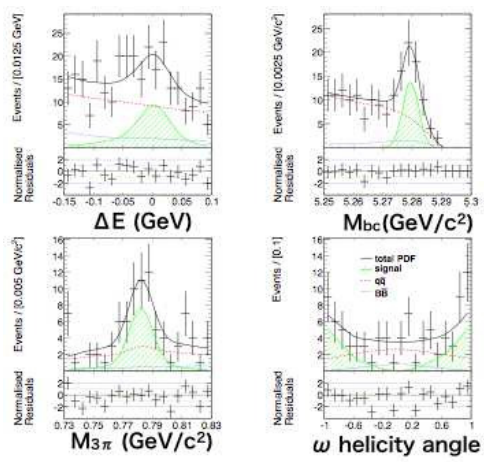

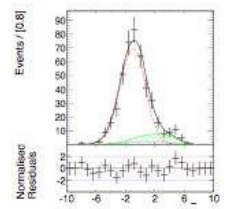

$q q$ suppression

- total PDF

- qव $\mathrm{BG}$

. $\mathrm{B} \overline{\mathrm{B}} \mathrm{BG}$

/ signal

Figure 1. Reconstructed variables of $B^{0} \rightarrow \omega K$ : $\Delta E$ (top left), $M_{\mathrm{bc}}$ (top middle), $q \bar{q}$ suppression (top right), invariant mass of $3 \pi$ (bottom left) and helicity angle (bottom right) of $\omega$ candidates. The solid lines show the fit result of signal extraction, dashed lines for $q \bar{q}$, dotted lines for $B \bar{B}$ background and shaded region for signal.

penguin diagram. To determine the $\phi_{2}$ without the extra-phase, several techniques are developed for data analysises in the $B$ decays. Using the isospin relations among the decay amplitudes of $B \rightarrow \pi \pi$ system, the extra-phase is determined with the four-fold ambiguity [7]. We have measured them in the $B^{0} \rightarrow \pi^{+} \pi^{-}$[8] and $B^{+} \rightarrow \pi^{+} \pi^{0}$ [9] precisely with the full data set. Preliminary result of $B^{0} \rightarrow \pi^{0} \pi^{0}$ is recently obtained with the full data set and new analysis technique using the ECL timing information to remove background. Figure 3 shows the distribution of reconstructed variables for the signal identification. We obtain the branching fraction $\mathcal{B}\left(B^{0} \rightarrow \pi^{0} \pi^{0}\right)=(0.90 \pm 0.12 \pm 0.10) \times 10^{-6}$ with the significance of $6.7 \sigma$ and the direct $C P$ asymmetry $A_{C P}=-0.054 \pm 0.086$. With this result, the constraint to the $\phi_{2}$ using the isospin relation in the $B \rightarrow \pi \pi$ system will be reevaluated.

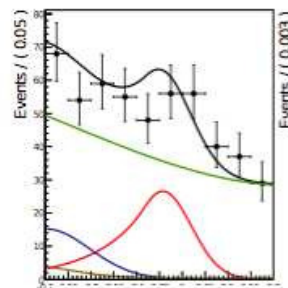

$\Delta \mathrm{E}(\mathrm{GeV})$

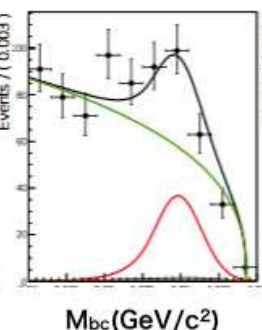

$\mathrm{Mbc}\left(\mathrm{GeV} / \mathrm{c}^{2}\right)$

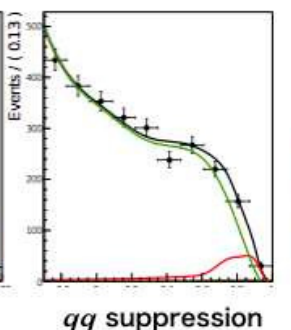

$q q$ suppression
Total PDF

Signal

qव

rare $\mathrm{B} \overline{\mathrm{B}}$

$\mathrm{B} \rightarrow \mathrm{p} \pi$

Figure 3. Reconstructed variables of $B^{0} \rightarrow \pi^{0} \pi^{0}: \Delta E$ (left) $M_{\mathrm{bc}}$ (center) and continuum suppression (right).

Non-zero $C P$ violation is expected from the contribution of new physics in decays induced by the $b \rightarrow s \gamma$ penguin transition such as the decay of $B \rightarrow X_{s} \gamma$, where the $X_{s}$ is a meson with a $s$-quark. In theoretical expectations, uncertainty arise due to the long-range strong interaction. However, it cancels 
when performing a combined analysis for the final-states of the $X_{s}$ and $X_{d}$ [10]. Figure 4 shows the distribution of the energy of the photon in the decay candidates that are reconstructed inclusively. We obtain the preliminary result $A_{C P}=(2.2 \pm 4.0 \pm 0.8) \times 10^{-2}$ for the signal enhanced region of $E_{\gamma}^{\mathrm{CM}}>2.2$ $\mathrm{GeV}$ where $E_{\gamma}^{\mathrm{CM}}$ is an energy of the $\gamma$ from the $B$ in center-of-mass (CM) system in the $e^{+} e^{-}$collision. This is consistent with zero which is expected from the SM.

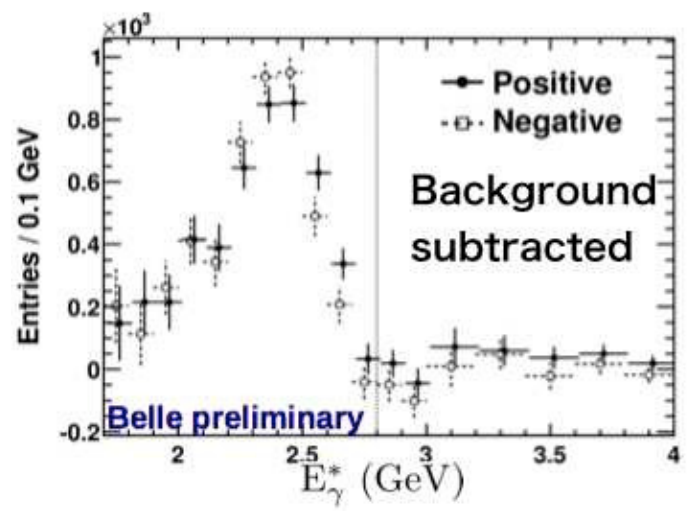

Figure 4. Energy distribution of $B^{0} \rightarrow X_{u, d} \gamma$ candidates. Filled circles with solid lines show that of the $B^{0} / B^{+}$ tagged candidates and open circles with dashed lines are that for the $B^{0} / B^{+}$tagged ones.

\section{Branching fraction measurements}

Signal from the new physics is also expected to be appeared in the decay rates, especially in the mode which is induced by the diagram with a loop.

Among such decay modes, the decay $B \rightarrow X_{s} \gamma$ is good probe to test the theoretical estimation with the next-next-leading-order (NNLO) and the branching fraction is expected to be $\mathcal{B}\left(B \rightarrow X_{s} \gamma\right)=$ $(3.15 \pm 0.23)^{-4}$ for $E_{\gamma}^{\mathrm{CM}}>1.6 \mathrm{GeV}$ [11]. To identify the $X_{s}$, there are several analysis approaches: full-exclusive in which single photon energy spectrum is observed, semi-inclusive in which as much as reconstructed decay mode of hadrons with $s$-quark are combined, and exclusive in which a specific decay mode such as $B \rightarrow K^{*} \gamma$ is reconstructed. Due to the QCD uncertainties in the hadronization in the initial- and final-states, a theoretical uncertainty becomes large in the exclusive approach. On the other hand, a signal purity becomes worse due to the background in the inclusive approaches. We recently update the result of the semi-inclusive analysis using $38 X_{S}$ decay modes in the invariant mass range of $0.6 \mathrm{GeV}<M_{X_{s}}<2.8 \mathrm{GeV} / c^{2}$ by combinations of the $K, \pi$ and $\eta$ which corresponds to about $70 \%$ of the total $X_{s}$ decays. Figure 5 shows the partial branching fraction as a function of the $X_{s}$ mass. From this data sample, we obtain the branching fraction $\mathcal{B}\left(B \rightarrow X_{s} \gamma\right)=(3.74 \pm 0.39) \times 10^{-4}$ and this is consistent with the SM expextation.

In charmless hadronic $B$ decays, loop appears in the penguin diagram with the first order of the decay process and its effect is appeared in the branching fraction, polarization, direct $C P$ violation and so on. We recently report the branching fraction measurements in $B^{+} \rightarrow \bar{K}^{*}(892)^{0} K^{*}(892)^{+}$induced by the $b \rightarrow d$ penguin and $B^{0} \rightarrow \eta^{\prime} K^{*}(892)^{0}$ induced by the $b \rightarrow s$ penguin.

In the $B^{+} \rightarrow \bar{K}^{*}(892)^{0} K^{*}(892)^{+}$decay, a longuitudinal polarization of the $B$ decay to two vector meson state $f_{\mathrm{L}}$ is also measured to validation of the $\mathrm{SM}$ in which $f_{\mathrm{L}} \simeq 1$ is expected from 


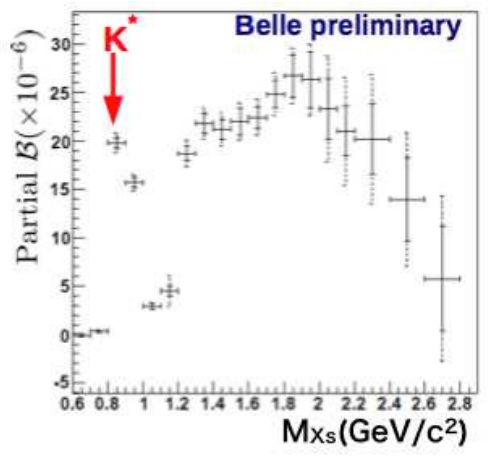

Figure 5. The partial branching fraction as a function of $M_{X_{s}}$ in $B^{0} \rightarrow X_{s} \gamma$ candidates in semi-inclusive reconstruction.

the QCD. Figure 6 shows the fit result to the seven-dimensional variables. From this, we obtained the branching fraction $\mathcal{B}\left(B^{+} \rightarrow \bar{K}^{*}(892)^{0} K^{*}(892)^{+}\right)=\left(0.77_{-0.30}^{+0.35} \pm 0.12\right) \times 10^{-6}$ and polarization $f_{\mathrm{L}}\left(B^{+} \rightarrow \bar{K}^{*}(892)^{0} K^{*}(892)^{+}\right)=1.06 \pm 0.30 \pm 0.14$, where the significance of the non-zero branching fraction is $2.7 \sigma$.

Total PDF Signal $q \bar{q}+B \bar{B}$ background Peaking background
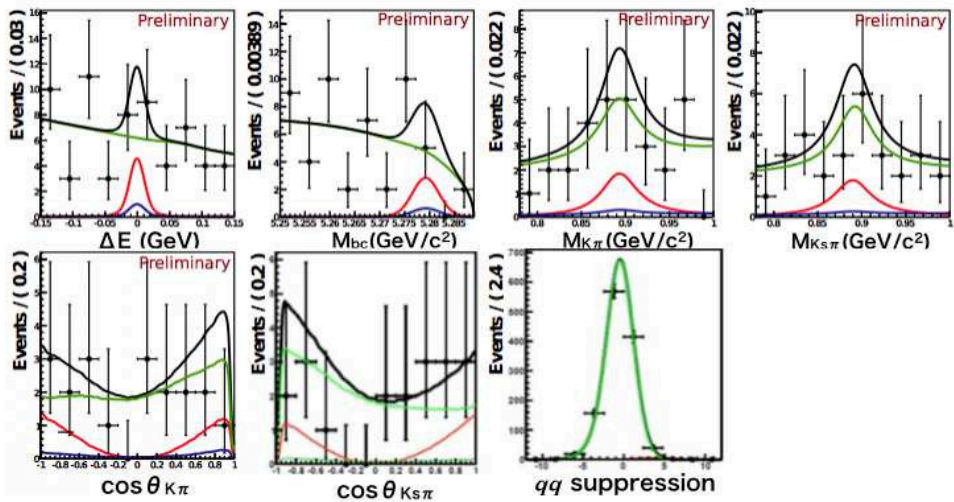

Figure 6. Reconstructed variables for $B^{+} \rightarrow \bar{K}^{*}(892)^{0} K^{*}(892)^{+}$identification, points with error bars is the data distribution and lines are the fit results: $\Delta E$ (top left), $M_{\mathrm{bc}}$ (top second from left), $M_{K \pi}$ (top third from left), $M_{K_{S} \pi}$ (top right), $\cos (K \pi)$ (bottom left), $\cos \left(K_{S} \pi\right)$ (bottom middle) and $q \bar{q}$ suppression (bottom right).

In the $B^{0} \rightarrow \eta^{\prime} K^{*}(892)^{0}$ decay, a large $C P$ violation is expected from the interference of $b \rightarrow s$ penguin and $b \rightarrow u$ tree diagrams and an evidence is seen in the $B^{0} \rightarrow \eta K^{0}$ decay, in which same interference is considered, in both of the Belle and BABAR $[12,13]$. We recently release the result of $B^{0} \rightarrow \eta^{\prime} K^{*}(892)^{0}$ decay. Figure 7 shows the fit result for the four-dimensional variables to extract the signal yield. From the extracted signal, we obtain the branching fraction $\mathcal{B}\left(B^{0} \rightarrow \eta^{\prime} K^{*}(892)^{0}\right)=$ 
$(2.6 \pm 0.7 \pm 0.2) \times 10^{-6}$ and direct $C P$ violation $A_{C P}\left(B^{0} \rightarrow \eta^{\prime} K^{*}(892)^{0}\right)=-0.22 \pm 0.29_{-0.03}^{+0.02}$, where the significance of the branching fraction measurement including the systematic uncertainty is 5.0 $\sigma$ [14].

All of the results of charmless hadronic $B$ decays are consistent with the SM expectations and world averages.
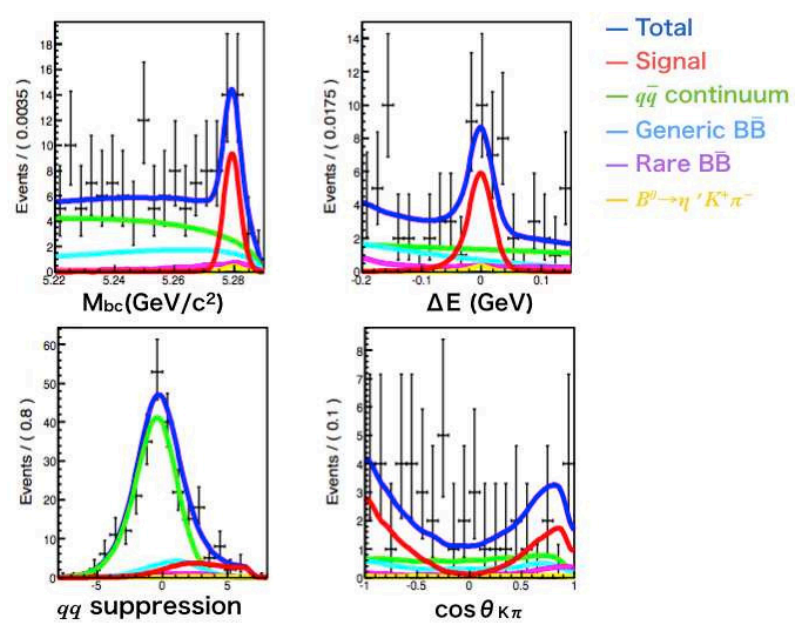

Figure 7. Reconstructed variables for $B^{0} \rightarrow \eta^{\prime} K^{*}(892)^{0}$ identification, points with error bars is the data distribution and lines are the fit results: $M_{\mathrm{bc}}$ (top left), $\Delta E$ (top right), $q \bar{q}$ suppression (bottom left) and $\cos (K \pi)$ (bottom right).

\section{CKM matrix elements}

In the semi-leptonic decay $B \rightarrow \bar{D}^{(*)} \ell v$ induced by the $b \rightarrow c \ell v$ tree diagram exchanging $W$ boson, a CKM matrix element $V_{c b}$ is measured from the differential decay width for kinematic variables $w$, inner product of the $B$ and $D$ velocities. However due to the initial- and final-state hadronization, measured variable includes a form factor. We recently update the result of semi-inclusive analysis in which $\bar{B}^{0} \rightarrow D^{+} \ell v$ with seven $D^{+}$decay modes and $B^{-} \rightarrow \bar{D}^{0} \ell v$ with eight $\bar{D}^{0}$ modes is reconstructed to reduce the uncertainty from the form factor. We measure the signal yields from subdivided data sample respected to the $w$ and measure $V_{c b}$ from its spectrum. Figure 8 shows the signal extraction in a $w$ range and spectrum of the signal yield as a function of the $w$. From the distribution, we obtained the results of $\eta_{\mathrm{EW}} \mathcal{G}(1)\left|V_{c b}\right|=42.63 \pm 0.96 \pm 1.39$ where $\eta_{\mathrm{EW}}$ is an electroweak correction and $\mathcal{G}(1)$ is a form factor. This is consistent with the world average of other measurements.

\section{Summary}

Using the full data sample accumulated in the Belle experiment which corresponds to the 772 million $B \bar{B}$, we have been investigating a signal of the new physics beyond the SM in various $B$ decays. We recently release the results of the measurements in $C P$ violation, branching fraction and so on. Most of the results are obtained with the best accuracy in the world but evidence of the new physics has not seen. We need further accuracy to access the new physics in higher energy scale from the view point 


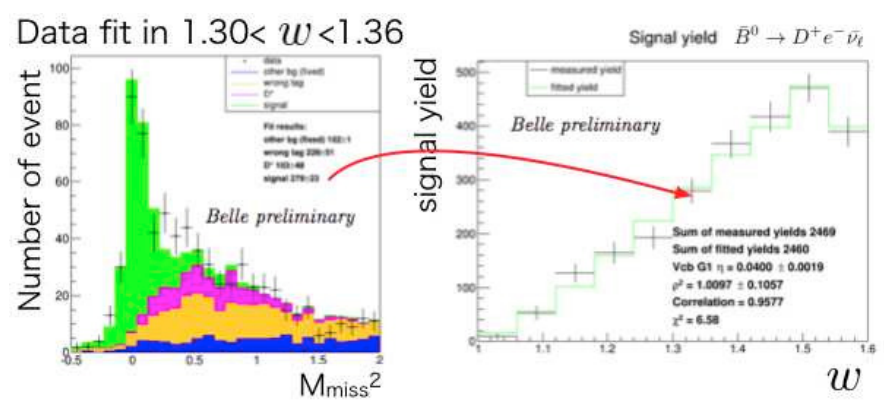

Figure 8. The $M_{\text {miss }}$ fit result of $B \rightarrow \bar{D}^{(*)} \ell v$ candidates in semi-inclusive reconstruction in range of $1.30<w<$ 1.36 (left) and signal yield from the fit as a function of $w$.

of a flavor physics and upgrade to the super-KEKB and Belle II is ongoing now aiming for 40 times higher luminosity and improvement of the detector performance [15].

\section{References}

[1] A. Abashian et al. (Belle Collaboration), Nucl. Instrum. Methods Phys. Res., Sect. A 479, 117 (2002); also, see the detector section in J. Brodzicka et al., Prog. Theor. Exp. Phys., 04D001 (2012).

[2] S. Kurokawa and E. Kikutani, Nucl. Instrum. Methods Phys. Res., Sect. A 499, 1 (2003), and other papers included in this volume; T. Abe et al., Prog. Theor. Exp. Phys., 03A001 (2013) and following articles up to 03A011.

[3] Z. Natkaniec et al. (Belle SVD2 Group), Nucl. Instrum. Methods Phys. Res., Sect. A 560, 1 (2006).

[4] M. Kobayashi and T. Maskawa, Prog. Theor. Phys. 49, 652 (1973).

[5] A. B. Carter and A. I. Sanda, Phys. Rev. D 23, 1567 (1981); I. I. Bigi and A. I. Sanda, Nucl. Phys. B 193, 85 (1981).

[6] V. Chobanova, J. Dalseno, C. Kiesling et al. (Belle Collaboration), Phys. Rev. D 90, 012002 (2014);

[7] M.Gronau and D. London, Phys. Rev. Lett. 65, 3381 (1990).

[8] J. Dalseno, K. Prothmann, C. Kiesling et al. (Belle Collaboration), Phys. Rev. D 88, 092003 (2013);

[9] Y.-T. Duh, T.-Y. Wu, P. Chang, G. B. Mohanty, Y. Unno, et al. (Belle Collaboration), Phys. Rev. D 87, 031103(R) (2013);

[10] T. Hurth, E. Lunghi and W. Porod, Nucl. Phys. B 704 (2005) 56.

[11] M.Misiak et al., Phys. Rev. Lett. 98022002 (2007).

[12] C.-T. Hoi et al. (Belle Collaboration), Phys. Rev. Lett. 108, 031801 (2012).

[13] B. Aubert et al. (BABAR Collaboration), Phys. Rev. D 80, 112002 (2009).

[14] S. Sato, Y.Yusa, G. Mohanty et al. (Belle Collaboration), Phys. Rev. D 90, 072009 (2014);

[15] T. Abe et al. (Belle II Collaboration), arXiv:1011.0352 [physics.ins-det]. 